Kalpa Publications in Engineering
Volume 3, 2020, Pages 42-48
Proceedings of International Sym-
posium on Applied Science 2019

\title{
Design And Manufacture Of A Device Using Transillumination And Scattering Near-Infrared Techniques For Detecting Dental Lesions
}

\author{
Hai Mien Pham Thi ${ }^{1}$, Khac Thinh Nguyen ${ }^{1}$, Phuoc An Truong Thi ${ }^{1}$, My \\ Nhat Truong Thi ${ }^{1}$, Minh Huong Nguyen Thi ${ }^{1}$ \\ ${ }^{1}$ Ho Chi Minh City University of Technology,VNUHCM \\ phamhaimien@hcmut.edu.vn
}

\begin{abstract}
In dentistry, near-infrared (NIR) technology has been studied for decades that is being applied for the detection of dental damages without using of ionizing radiation. Based on the interaction between dental tissue and NIR light such as transmission, absorption and scattering, the teeth structure and dental lesions can be observed by NIR image. The aim of this study was to build the dental diagnostic device using NIR light at $850-\mathrm{nm}$ wavelength for detecting the early and hidden dental damages, that are not observed by $\mathrm{X}$-rays. According to the various types of teeth samples, the transillumination and the scattering methods were designed and applied for capturing the teeth structures. The transillumination technique was used for thin teeth such as incisor and canine while the scattering system for observing occlusal surface. The results show that the areas suspected to be the demineralized enamel are distinctly distinguished from the surrounding sound tissues and especially, the stain and pigmentation don't appear in NIR image. The designed device meets some requirements such as simple setup, safety and affordable price for the purpose of replacement of imported equipment.
\end{abstract}

\section{Introduction}

Many researches show that the status of cavities or lesions of the teeth is increasing all over the world in general and Viet Nam in particular. Surveys conducted by the hospital with Adelaide University show an alarming rate of dental problems among the country's young: $85 \%$ of children aged 6 to 8 have decayed milk teeth, while $54,6 \%$ in the $9-11$ are group with permanent tooth decay. The number of people suffering dental caries increases in accordance with their age and the severity. Over $90 \%$ of Vietnamese suffer from dental disease according to the National Hospital of OdontoStomatology. The incidence of dental lesions is increasing and common in both children and adults, the 
main reason is due to poor oral hygiene, diet and no periodic dental examinations. Therefore, the day of Oral Health worldwide March 20 every year is necessary.

Nowadays, there are many methods of tooth decay such as X-rays, measuring electrical conductivity of teeth, fluorescence methods, etc. X-Rays is a reliable method that dentists use to evaluate related dental structures, which is considered "Gold Standard" to check dental structure and defects in dentistry. However, X-rays is also limited, it affects the health of patients and dentists [1],[2]. In addition, it also requires the photographer to understand the shooting techniques because of the effect on diagnosis.

In recent year, near-infrared (NIR) method was born as a new research, this method is not only for observing the structure of teeth, but also can simultaneously observe, monitor and evaluate new lesions, defects in the teeth that the naked eye hard to detect, that is being investigated for the detection of early damages without using of ionizing radiation [3],[4]. NIR method shows tooth structure image detected by NIR camera. The created images are based on the optical properties of the tooth due to the transmission, absorption and scattering of dental tissues in NIR wavelength.

The damaged tooth structure is mostly demineralized enamel in which mineral density is reduced. Demineralization creates gaps scattering strongly in near infrared at the wall of gaps [5]. For demineralization, the scattering coefficient of demineralized enamel increases by $1-2$ orders of magnitude at wavelength $1300 \mathrm{~nm}$ [6]. Because of the optical properties difference of sound enamel and demineralized enamel, the contrast between sound enamel and demineralized enamel in the near infrared image is high. In X-rays method, the contrast between sound enamel and demineralized enamel isn't clear [4], [7].

The aim of this study was to build the optical system observing tooth structure in wavelength $850 \mathrm{~nm}$. Many researches have indicated that wavelength $1300 \mathrm{~nm}$ is more effective than $850 \mathrm{~nm}$ due to the higher contrast of images at $1300 \mathrm{~nm}$ but performance is so hard because of the limited equipment (LED and camera). Therefore, the system used 850-nm wavelength whose extinction is lower than $40 \mathrm{~cm}-1$ in enamel and absorption of water is negligible [4]. 850-nm LED and NIR camera are available to get with affordable cost. In this study, the transillumination and the scattering methods were used to design the device for capturing the approximal and occlusal images of teeth.

\section{Design - Build process}

\subsection{The principle of the device}

In the scattering method, the optical system was designed with two symmetrical 850 - nm LEDs located next to two opposite sides of the tooth and one intraoral NIR camera placed above the occlusal surface of the tooth (fig. 1A). The distance between the camera and the tooth was adjusted to suit each tooth sample $(\sim 2 \mathrm{~cm})$ in order to record the most qualified images. Because the absorption coefficients are exceptionally small in visible and NIR light, while scattering is strong in visible light and weak in NIR, enamel is translucent in the near infrared light.

For dentin, the absorption coefficient depends upon the wavelength in the visible region with a value of $\mu \mathrm{a} \sim 4 \mathrm{~cm}-1$, while the scattering is strong throughout the visible and NIR regions [8]. Therefore, in this method, LED light enters the teeth just above the gumline, penetrates the enamel layer and is scattered by the dentin [9]. As a result, the scattered light migrates upward to the occlusal surface of the tooth, allowing for observing lesions on occlusal surface and side surface of tooth. 
Design and manufacture of a device using transillumination and scattering near-infrared ... Thi et al.

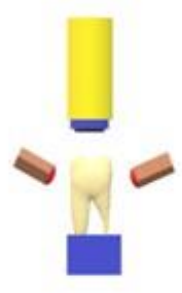

A)

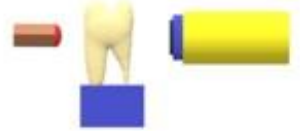

B)

Figure 1: Schematic diagrams of scattering (A) and transillumination (B) systems.

In the transillumination method, the optical system consists of a 850 - nm LED and an intraoral camera located next to opposite sides of the tooth (Fig. 1B). The camera is placed at a fixed distance from the tooth $(\sim 2 \mathrm{~cm})$ while the LED is in the direct contact with side surface of tooth. The camera and the LED were set coaxially with the tooth sample. This arrangement allows light from the LED to go through both enamel and dentin. The transillumination method suits for thin teeth such as incisor and canine.

In this study, we designed a device that combines two above mentioned scattering and transillumination techniques with the ability to provide an overview of the tooth including approximal and occlusal lesions. The device powered by USB port of computer consists of three function blocks: the camera block, the LEDs block and the control circuit block (Fig. 2).

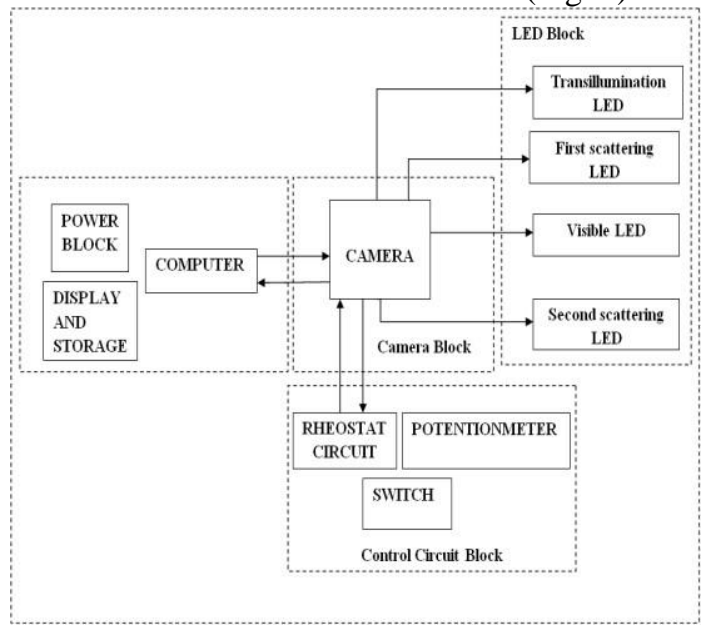

Figure 2: Schematic diagrams of scattering (A) and transillumination

\subsection{Design of the device}

\subsubsection{LEDs block}

The equipment for detection of dental caries is a system that consists of two types of LEDs: three 850 - nm LEDs for capturing NIR images and six white LEDs for visible observation.

As mentioned above, the wavelength of $1300 \mathrm{~nm}$ is more effective than $850 \mathrm{~nm}$ due to the higher contrast of images at $1300 \mathrm{~nm}$ but performance was challenging because of the limited equipment (LED and camera). Therefore, the system used a 850- $\mathrm{nm}$ wavelength of which the extinction is lower in enamel and absorption of water is negligible. 850-nm LED and NIR camera are available at an 
Design and manufacture of a device using transillumination and scattering near-infrared ... Thi et al.

affordable cost. Technical specifications of 850 - nm LEDs: consumption power $1 \mathrm{~W}$, input voltage 1.5 to $1.8 \mathrm{~V}$, forward current $500 \mathrm{~mA}$, lumen efficiency $42 \mathrm{~lm} / \mathrm{W}$ and emitting angle $120^{\circ}$.

The original camera was integrated six white LEDs surrounding its head, helping observe and capture visible images. The white LEDs have consumption power $0.7 \mathrm{~W} / 3 \mathrm{pcs}$, input voltage $12 \mathrm{~V} / 6 \mathrm{pcs}$, and lumen efficiency $72 \mathrm{~lm} / \mathrm{W}$.

The scattering method

In regard to the scattering method, two 850-nm LEDs were placed into the rectangular boxes to create a fixed contact plane between NIR LEDs and the tooth surface. Two boxes and NIR camera were attached together by the axes forming a complete optical system.

It is worth noting that, if two LEDs blocks were parallel to each other (Fig. 3A), the light intensity would be immensely strong leading to the glare of the images. To overcome that obstacle, the distance between two blocks containing LEDs was designed smaller than the thickness of the tooth sample. Due to the plasticity of material of the boxes, when the tooth is inserted into the space between boxes, it will expand. As a result, the axes of the two boxes will create an $\alpha$-angle to the axis of the camera (Fig. 3B).Due to the short focal length of the camera, the distance from the tooth sample to the camera was fixed at $25 \mathrm{~mm}$.
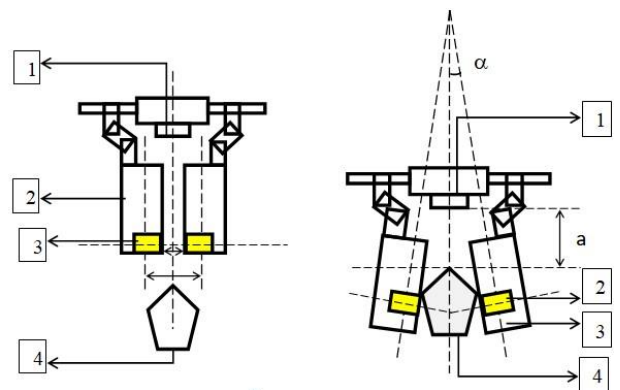

Figure 3: Design of the LEDs block: 1 - Camera, 2 - LEDs boxes, 3 - 850-nm LEDs, 4 - Tooth sample.

\section{The transillumination method}

Unlike the scattering method, the transillumination system consists of the unique NIR LED placed as close as possible to one side of the tooth sample, the opposite side of which was faced the camera (Fig. 4). The other components of this system are similar to the above mentioned scattering technique.

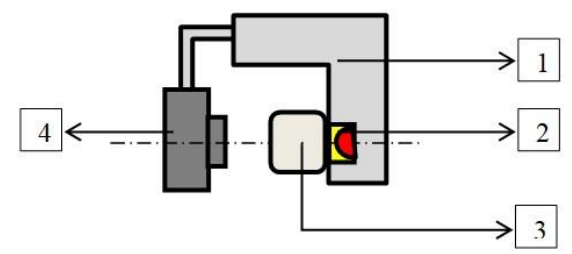

Figure 4: Design of the LEDs block: 1 - LED box, 2 - 850nm LED, 3 - Tooth sample, 4 -Camera.

\subsubsection{Camera}

An intraoral camera connected to a computer with a USB cable 2.0 was used for capturing images. Due to the space limitation in the oral cavity, the camera has a short focal length and the appropriate size. Technical specifications of the chosen camera: sensor type CMOS, resolution $2 \mathrm{Mpx}$, image rate $25 \mathrm{fps}$, focal length 20 to $30 \mathrm{~mm}$, input voltage $5 \mathrm{~V}$, temperature range $10-75^{\circ} \mathrm{C}$, and outer dimensions of $17 \mathrm{~cm}$ (height) $2 \mathrm{~cm}$ (width) $1 \mathrm{~cm}$ (depth). 
Design and manufacture of a device using transillumination and scattering near-infrared ... Thi et al.

\subsubsection{Control circuit block}

Power of light sources is one of the most important factors affecting the NIR image quality. If the LEDs power is too high, the captured images will be dazzling and no details of teeth can be seen. For that request, a potentiometer is necessary to adjust the light intensity of LEDs.

Technical specifications of the potentiometer: CT6R2 rotating type, two polar pairs, three contact points, voltage ranting DC $30 \mathrm{~V} 1 \mathrm{~A}$.A three - mode switch (ON-OFF-ON) was equipped for converting between two capturing modes, making it easy to compare the infrared and visible images.

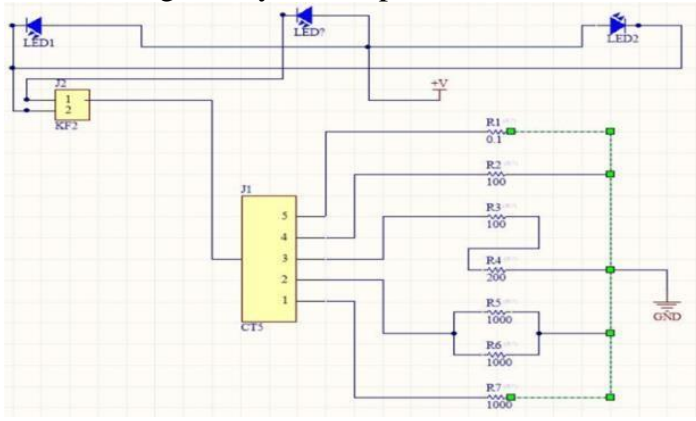

Figure 5: Diagram of control circuit block.

\subsection{Build process}

The build process of the device includes the following steps: 1) Design the device model using Solidworks software; 2) 3D printing process.

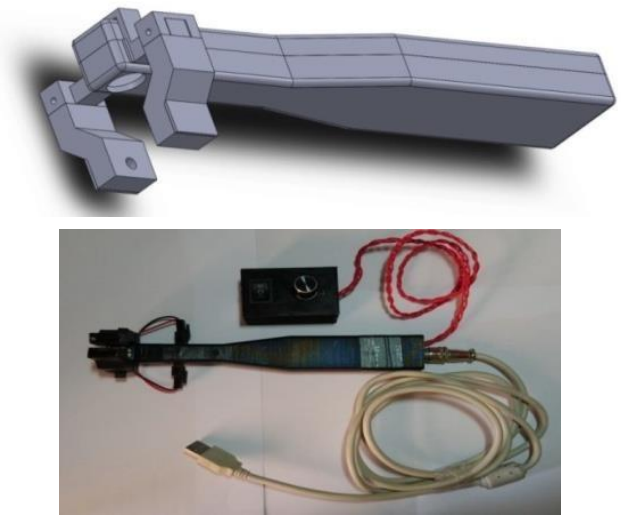

Figure 6: 3D - design and appearance of scattering device.

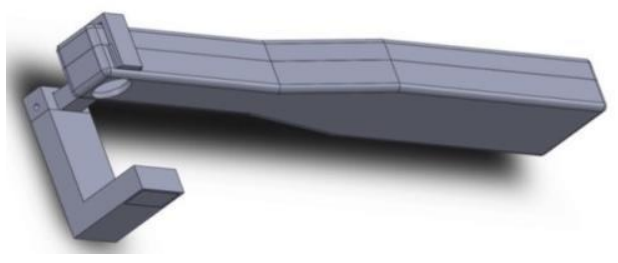


Design and manufacture of a device using transillumination and scattering near-infrared ... Thi et al.

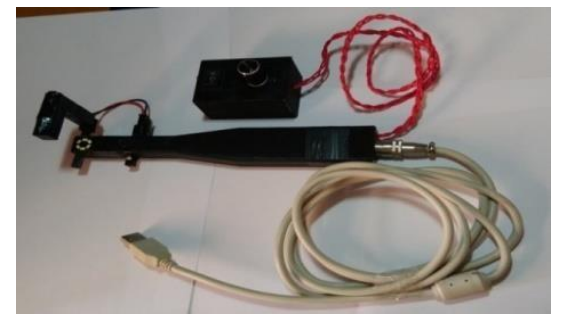

Figure 7: 3D - design and appearance of transillumination device.

\section{Test of device}

There are many causes of tooth decay corresponding to different types of lesions which are often overlooked in clinical observation, such as demineralisation, fluorosis or white spot lesions. Substantial research has showed that under NIR light many lesions appear with high contrast because the scattering coeffcient of damaged tissue is lower than that of healthy enamel [8]. Althought X-rays is considered gold standard to check dental structure and defects in dentistry, but it is unable to detect white spot lesions at early stages [1].

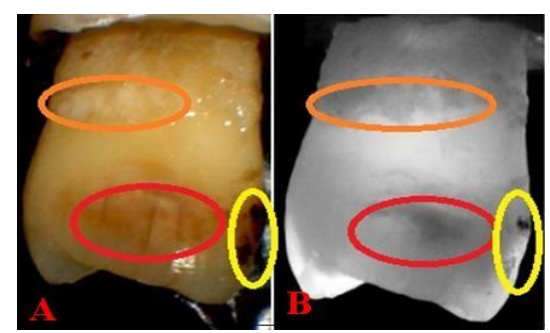

Figure 8: Sample 1 under visible light (A), NIR light (B).

External lesions of the teeth are often overlooked during clinical examination, because these lesions are limited in vision. Figure 8A shows a tooth sample under visible light with some suspected lesions such as white spot or dental plaque (circled areas). In this case, the clinical observation is difficult to determine the exact location of the lesions. Nevertheless, these lesions are more apparent on the NIR image (Figure $8 \mathrm{~B}$ ) with high contrast so that it is easy to distinguish the lesions from the surrounding sound enamel.

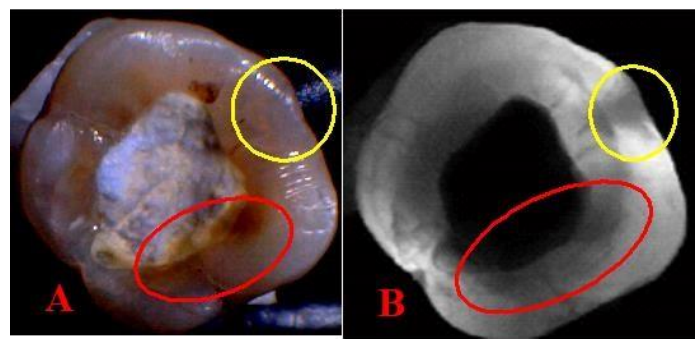

Figure 9: Sample 1 under visible light (A), NIR light (B)

Figure 9A represents a tooth sample with a filling on the occlusal surface, which shows no signs of injury under visible light. However, in figure 9B, under infrared light, the enamel around the filling appears with some dark areas (circled areas), which are likely to be linked to the existence of the recurrent caries under the filling.

Besides, the device offer the clarity of images and relatively high resolution $1280 \mathrm{x} 960$, which helps distinguish sound enamel and demineralization. 
Design and manufacture of a device using transillumination and scattering near-infrared ... Thi et al.

\section{Conclusion}

In order to design and build a portable device, which can effectively support clinicians in detecting the early dental lesions, in this study, two different device models with transillumination and scattering techniques using 850-nm LED were designed and built up. These devices have met numerous criteria such as simple setup, flexibility, safety and affordable price. The test result indicated that the captured infrared images have good contrast for distinguishing between sound teeth and dental lesions under enamel layer, showing the possibility of applying the NIR techniques in the development of a specialized dental tool without the use of ionizing radiation. In the future, the hardware of the device requires further improvement and an image processing software will be developed to increase the image quality for in vivo application.

\section{References}

Zangooei B. Dental caries diagnostic methods, DJH 2010; 2(1), 1-12.

Mirela M-T, et al. Effectiveness of near-infrared transillumination in early caries diagnosis, Biotechnology and Biotechnological Equipment 2016; 30(6), 1207-1211.

Bussaneli DG, Restrepo M, et al. Assessment of new infrared laser transillumination technology $(808 \mathrm{~nm})$ for the detection of occlusal caries-an in vitro study, Lasers Med Sci 2015; 30(7), 1873-1879.

Christopher MB, Patara N and Daniel F. Imaging of occlusal dental caries (decay) with near-IR light at 1310- nm, Optics Express 2005; 13(2), 573-582.

Pham THM, Le PD, Ta KT. Attempting nearinfrared transillumination imaging with sample intrumentation for studying dental lessions, Physical Science 2019; 42-43.

Simon JC, et al. Near-IR transillumination and reflectance imaging at 1,300 nm and 1,5001,700 nm for in vivo caries detectioc, Lasers in Surgery and Medicine 2016; 48(9), 828-836.

Robert SJ, Daniel F, et al. Near-infrared transillumination at 1310-nm for the imaging of early dental decay, Optics Express 2003; 11(18), 2258-2265.

Daniel F, Michal S, Darling CL. Near-infrared imaging of dental decay at 1,310 nm, J. Laser Dent 2010; 18(1), 8-16.

Krista AH, Daniel F, Darling CL. Near-infrared imaging of developmental defects in dental enamel, Journal of Biomedical Optics 2008; 13(4), 044011. 